\title{
A library of monoalkenylsilsesquioxanes as potential comonomers for synthesis of hybrid materials
}

\author{
Katarzyna Mituła1,2 $\cdot$ Michał Dutkiewicz $^{2} \cdot$ Beata Dudziec $^{1,2}$ (D) Bogdan Marciniec ${ }^{1,2} \cdot$ Krystyna Czaja $^{3}$
}

Received: 25 October 2017 / Accepted: 24 February 2018/Published online: 7 March 2018

(C) The Author(s) 2018. This article is an open access publication

\begin{abstract}
In this paper we demonstrate a facile and efficient synthesis of monoalkenylsubstituted POSS compounds, i.e., $\mathrm{R}_{7}\left(\mathrm{Si}_{8} \mathrm{O}_{12}\right)$ alkenyl and $\mathrm{R}_{7}\left(\mathrm{Si}_{8} \mathrm{O}_{12}\right)\left(\mathrm{OSiMe}_{2}\right)$-alkenyl with a functional alkenyl group of chain length (from vinyl to dec-9-enyl, with or without additional $-\mathrm{OSiMe}_{2}-$ spacer between the silsesquioxane core and alkenyl moiety) and with five types of inert groups (Et, iBu, iOc, $\mathrm{Cy}, \mathrm{Ph}$ ) at the POSS core. These compounds constitute a library of monosubstituted POSS systems, including thorough spectroscopic and thermal characteristics. These findings could be especially useful for material chemists applying the compounds as modifiers or comonomers for copolymerization reaction, e.g., with olefins.
\end{abstract}

\section{Graphical Abstract}

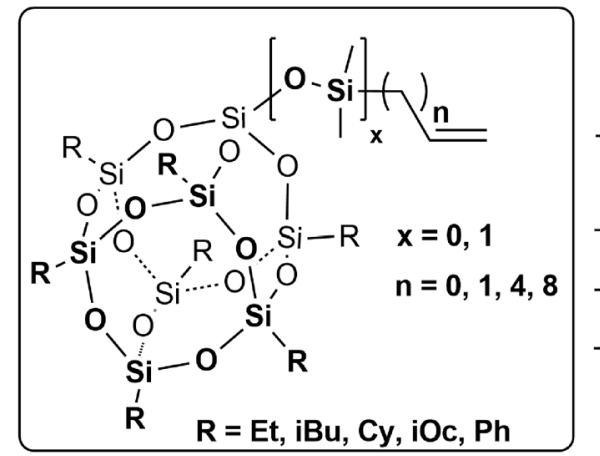

Electronic supplementary material The online version of this article (https://doi.org/10.1007/s10973-018-7121-2) contains supplementary material, which is available to authorized users.

\section{Beata Dudziec}

beata.dudziec@gmail.com

$\triangle$ Bogdan Marciniec

Bogdan.Marciniec@amu.edu.pl

1 Faculty of Chemistry, Adam Mickiewicz University in Poznan, Umultowska 89B, 61-614 Poznan, Poland

2 Centre for Advanced Technologies, Adam Mickiewicz University in Poznan, Umultowska 89C, 61-614 Poznan, Poland

3 Faculty of Chemistry, Opole University, Oleska 48, 45-040 Opole, Poland
- simple synthetic routes to obtain a library of 40 compounds

- different alkenyl chain length and inert group

- high isolated yields up to $98 \%$

- verification of thermal properties dependence on alkenyl functional and inert substituents

Keywords Alkenylsubstituted silsesquioxanes · Polyhedral oligomeric silsesquioxanes - Thermal properties - Thermal stability

\section{Introduction}

The unique structures of polyhedral silsesquioxanes (POSS) of the general formula $\left(\mathrm{RSiO}_{3 / 2}\right)_{\mathrm{n}}$ have attracted widespread attention as precursors and components of a variety of inorganic/organic hybrid materials, as well as their application in optics, catalysis, and electronics, etc. $[1,2]$. They have also attracted the increasing attention of material scientists investigating the structure-property relationship of polymer/POSS composites. As nanosized building blocks, they have been widely studied because of 
their behavior at the nanoscale $[3,4]$. There are reports indicating the great interest of material chemists in silsesquioxanes with one or more functionalities and their potential use in the modification of various types of polymers. As is known, there are a few possibilities to introduce functional silsesquioxane into the polymer matrix. This is related to the amount and type of the functional groups (FG) attached to the POSS core and whether they are reactive or inert $[1,2,5]$. When the POSS molecules contain only inert groups, it is possible to introduce them into the already existing polymer matrix by physical mixing or performing a polymerization of the comonomer in the presence of the abovementioned POSS compound (POSS is, in effect, "blended" into the polymer matrix). Another method is to perform copolymerization with the organic comonomer and silsesquioxane containing the same or similar FG. As far as copolymerization is concerned it also matters how many FG there are at the POSS core and if they are all capable of reacting with the comonomer. In this way, silsesquioxane may be incorporated as a pendant group or cross-linker, respectively. Finally, it is also feasible to perform direct cross-linking of POSS units possessing only reactive FG, without an additional copolymer, to form a three-dimensional network. As a result, POSS species can be dispersed or aggregated/agglomerated together as either crystalline or even amorphous phase. Taking this into consideration, the resulting materials of a hybrid, inorganic-organic nature may exhibit interesting and improved physicochemical parameters, i.e., thermal, mechanical and optical $[3,6,7]$. These options are visualized in Fig. 1.

The monofunctionalized $\mathrm{T}_{8}$-silsesquioxanes are of specific importance as they can interact with the polymeric matrix in a grafting process or, alternatively, play the role of a comonomer in a copolymerization (or homopolymerization [8]) reaction with organic mers, resulting in a polymer with pendant POSS units [3, 9-14]. However, the properties in resulting composites may significantly differ, due to the distinct amount of POSS pendant moieties in the organic matrix. As trisilanols are commercially available, it is feasible to introduce a great variety of FG to the POSS core. Resulting compounds may be used as modifiers of a wide range of organic polymers, e.g., polyolefins, polyethers, polyamides, polyimides, polystyrenes, polyacrylates, polyurethanes, epoxy resins or carbon-based materials. [3, 15]. Because of their advantageous properties, polyolefins, such as PE, PP and PS, are widely used in a number of areas (from industry to everyday life). However, the possibilities of their modification (especially PE and PP) with POSS units can be limited and concern either physical blending (POSS with inert groups) or their initial preparation, i.e., irradiation or temperature initiation to create active sites at the polymer matrix, which are able to be grafted with FG of modifiers (usually via radical mechanism) $[12,16]$. For PS, it is also likely to functionalize their phenyl ring using, e.g., Friedel-Crafts reaction [17]. Unfortunately, for methods involving initial polymer preparation, partial degradation of the polymer may be observed. Hence, reports have been published on the preparation of polyolefin/POSS composites (containing mainly PE, PP and PS) via direct copolymerization of an olefin monomer with a functionalized POSS as a comonomer (in the presence of variety of catalytic systems including Ziegler-Natta, metallocene or post-metallocene complexes) [11, 18-29]. As is known, the resulting composites exhibit enhanced physicochemical properties (thermal, mechanical, morphological, rheological, etc.) to the parent polymers $[3,6,12]$.

Herein, we present a library of monofunctionalized silsesquioxanes with a different functional alkenyl group of diverse chain length (from vinyl to dec-9-enyl, with or without an additional $-\mathrm{OSiMe}_{2}-$ spacer between the silsesquioxane core and alkenyl moiety) and with five types of inert groups (Et, iBu, iOc, $\mathrm{Cy}, \mathrm{Ph}$ ) at the POSS core. This paper reports on their synthetic procedures involving condensation and/or hydrosilylation reaction, as well as a thorough spectroscopic and thermal characterization. This provides the opportunity to discuss not only the synthetic
Fig. 1 Possible organic polymer _POSS architectures
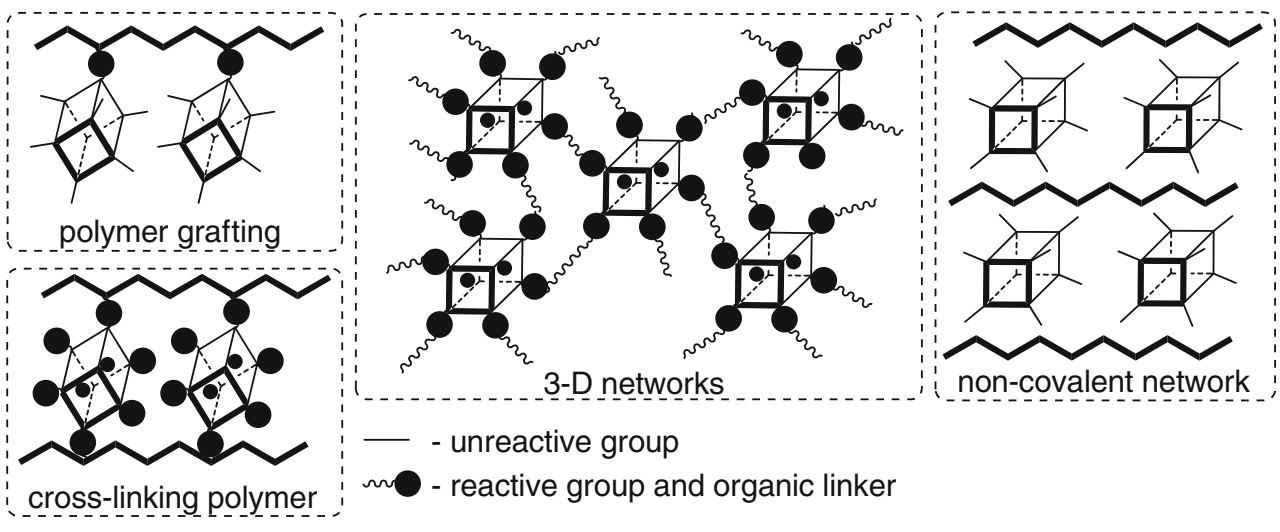
aspects of preparation of this large group (40 compounds) of monoalkenylfunctionalized silsesquioxanes (most of which are not found in the existing literature), but also the issues connected with their thermal resistance and possible degradation paths. Elaboration of efficient synthetic methodology for the abovementioned POSS-based systems would be particularly important for further application of these compounds as polymers modifiers or comonomers for copolymerization reaction with olefins.

\section{Experimental}

All syntheses were conducted under argon atmosphere using standard Schlenk-line and vacuum techniques. ${ }^{1} \mathrm{H}$, ${ }^{13} \mathrm{C}$ and ${ }^{29} \mathrm{Si}$ NMR spectra were recorded using Brucker Ultrashield 300 and $400 \mathrm{MHz}$ spectrometers, using $\mathrm{CDCl}_{3}$ as a solvent. Chemical shifts are reported in ppm with reference to the residual solvent $\left(\mathrm{CHCl}_{3}\right)$ peaks for ${ }^{1} \mathrm{H}$ and ${ }^{13} \mathrm{C}$ and to TMS for ${ }^{29} \mathrm{Si}$. FT-IR spectra were recorded on a Bruker Tensor 27 Fourier transform spectrophotometer equipped with a SPECAC Golden Gate, diamond ATR unit with a resolution of $2 \mathrm{~cm}^{-1}$. GC analysis was performed on a Bruker 430 gas chromatograph equipped with a TCD detector and capillary column megabore HP-130m (Hewlett-Packard). TLC was conducted on TLC-sheets coated with $0.2 \mathrm{~mm}$ thick silica gel with fluorescent indicator $\mathrm{UV}_{254}$. Column chromatography was performed on silica gel 60 (70-230 mesh) using hexane/DCM. MALDI-TOF mass spectra were recorded on a UltrafleXtreme mass spectrometer (Bruker Daltonics), equipped with a SmartBeam II laser $(355 \mathrm{~nm})$ in $500-4000 \mathrm{~m} / \mathrm{z}$ range. 2,5-Dihydroxybenzoic acid (DHB, Bruker Daltonics, Bremen, Germany) served as the matrix and was prepared in TA30 solvent (30:70 v/v acetonitrile: 0.1\% TFA in water) at a concentration of $20 \mathrm{mg} \mathrm{cm}^{-3}$. High-resolution mass spectrometry analyses were performed using a Synapt G2-S mass spectrometer (Waters) equipped with an electrospray ion source and quadrupole-time-of-flight mass analyzer. Methanol was used as a solvent. The measurement was taken in positive ion mode with a desolvation gas flow of $600 \mathrm{~L} \mathrm{~h}^{-1}$ and capillary voltage set to $4500 \mathrm{~V}$, with the flow rate $100 \mu \mathrm{L} \mathrm{m^{-1 }}$. The EIMS analysis was performed using a Bruker $320 \mathrm{MS} / 450 \mathrm{GC}$. TGA analyses were performed using a TGA4000 (PerkinElmer) thermal gravimetric analyzer. The measurements were conducted in nitrogen atmosphere (flow of $20 \mathrm{~cm}^{-3} \mathrm{~min}^{-1}$ ), in a temperature range $30-1000^{\circ} \mathrm{C}$, and heating rate $-10^{\circ} \mathrm{C} \mathrm{min}^{-1}$.

\section{Materials}

The chemicals were purchased from the following sources: silicon tetrachloride, trichlorosilane, chlorodimethylsilane, trichlorovinylsilane, allyltrichlorosilane, chloro(dimethyl) vinylsilane, allyl(chloro)-dimethylsilane, 1,5-hexadiene, Karstedt's catalyst (platinum(0)-1,3-divinyl-1,1,3,3-tetramethyldisiloxane complex $[\mathrm{Pt}(\mathrm{dvs})]$, solution in xylene with w2\% of Pt), calcium hydride, sodium hydride, ammonium chloride, lithium aluminum hydride (LAH), magnesium sulfate, triethylamine, $N, N$-diisopropylethylamine, toluene, tetrahydrofuran (THF), diethyl ether, methanol, $n$-hexane, $n$-pentane, dichloromethane (DCM), chloroform-d, molecular sieves type $4 \AA$ from SigmaAldrich. 1,9-decadiene from TCI. $\mathrm{R}_{7}\left(\mathrm{Si}_{7} \mathrm{O}_{9}\right)(\mathrm{OH})_{3}$ (trisilanol POSS; R = Cy, Et, iBu, iOc, Ph) from Hybrid Plastic. Silica gel-MN-Kieselgel 60 from Fluka Chemie AG. TLCsheets from Macherey-Nagel. Sartorius Minisart syringe filters (PTFE, $0.2 \mu \mathrm{m}$ ) from Sartorius AG.

\section{Synthetic procedures}

\section{Preparation of monosilanolPOSS (1-30H)- $\mathrm{R}_{\mathbf{7}}\left(\mathrm{Si}_{\mathbf{8}} \mathrm{O}_{12}\right)(\mathrm{OH})(\mathrm{R}=\mathrm{Et}, \mathrm{Ph})$}

\section{An example for alkyl inert group $(\mathrm{R}=\mathrm{Et})$}

$\mathrm{Et}_{7}\left(\mathrm{Si}_{7} \mathrm{O}_{9}\right)(\mathrm{OH})_{3}(\mathbf{1}-\mathbf{E t}-\mathbf{3 O H})\left(5.25 \mathrm{~g}, 8.8 \times 10^{3} \mathrm{~mol}\right)$, anhydrous THF $\left(100 \mathrm{~cm}^{-3}\right)$ and $\mathrm{Et}_{3} \mathrm{~N}\left(1.85 \mathrm{~cm}^{-3}\right.$, $\left.13.0 \times 10^{3} \mathrm{~mol}\right)$ were added to a two-neck, round-bottom flask equipped with a magnetic stirrer. The reaction mixture was placed in an ice-water bath and purged with argon, after which silicon tetrachloride $\left(1.06 \mathrm{~cm}^{-3}, 9.3 \times 10^{3} \mathrm{~mol}\right)$ was added. The reaction was carried out for $24 \mathrm{~h}$ at room temperature. Next, the insoluble solid of triethylammonium chloride was removed by filtration on a glass frit. The precipitate was washed with THF $(3 \times 5 \mathrm{~mL})$, and the solvent was evaporated. The residue left after evaporation was dissolved in a mixture of THF $\left(50 \mathrm{~cm}^{-3}\right)$ and water $\left(10 \mathrm{~cm}^{-3}\right)$ and heated at $65^{\circ} \mathrm{C}$ for $24 \mathrm{~h}$. After this time, the THF was evaporated and the remains were extracted with n-hexane $\left(100 \mathrm{~cm}^{-3}\right)$, water and brine. The organic phase was collected and dried with $\mathrm{MgSO}_{4}$. Evaporation gave the analytically pure product 1-Et-OH in the form of a white powder (5.45 g, 97\% yield) [30].

\section{An example for phenyl inert group $(\mathrm{R}=\mathrm{Ph})$}

$\mathrm{Ph}_{7}\left(\mathrm{Si}_{7} \mathrm{O}_{9}\right)(\mathrm{OH})_{3}(\mathbf{1 - P h}-3 \mathrm{OH})\left(1.14 \mathrm{~g}, 1.23 \times 10^{3} \mathrm{~mol}\right)$, anhydrous THF $\left(80 \mathrm{~cm}^{-3}\right)$ and $\operatorname{EtN}(\mathrm{iPr})_{2}\left(1.07 \mathrm{~cm}^{-3}\right.$, $\left.6.14 \times 10^{3} \mathrm{~mol}\right)$ were added to a two-neck round-bottom 
flask equipped with a magnetic stirrer. The reaction mixture was placed in an ice-water bath and purged with highly pure argon. Next, silicon tetrachloride $\left(0.14 \mathrm{~cm}^{-3}\right.$, $1.24 \times 10^{3} \mathrm{~mol}$ ) was added. The reaction was carried out for $24 \mathrm{~h}$ at room temperature. The THF was evaporated from the reaction mixture. After that, diethyl ether $\left(50 \mathrm{~cm}^{-3}\right)$ was added. The mixture was then extracted with water and brine. The organic phase was dried with $\mathrm{MgSO}_{4}$. Evaporation gave the analytically pure product as a white powder: $1.12 \mathrm{~g}$ of 1-Ph-OH with $94 \%$ yield.

\section{General procedure for preparation of alkenyl POSS compounds (3 and 3-OSi) (R7POSS-alkenyl and $\mathrm{R}_{7} \mathrm{POSS}-\mathrm{OMe}_{2} \mathrm{Si}$-alkenyl) via condensation reaction}

\section{An example for synthesis of $\mathrm{iBu}_{7} \mathrm{POSS}-\mathrm{Vi}(3-\mathrm{BBu}-\mathrm{Vi})$}

$\mathrm{iBu}_{7}\left(\mathrm{Si}_{7} \mathrm{O}_{9}\right)(\mathrm{OH})_{3}(\mathbf{1}-\mathbf{i B u}-\mathbf{3 O H})\left(1.00 \mathrm{~g}, 1.27 \times 10^{3} \mathrm{~mol}\right)$, anhydrous THF $\left(15 \mathrm{~cm}^{-3}\right)$ and $\mathrm{Et}_{3} \mathrm{~N}\left(0.64 \mathrm{~cm}^{-3}\right.$, $\left.4.56 \times 10^{3} \mathrm{~mol}\right)$ were added to a two-neck round-bottom flask equipped with a magnetic stirrer. The reaction mixture was placed in an ice-water bath and purged with highly pure argon. Next, trichlorovinylsilane $\left(0.16 \mathrm{~cm}^{-3}\right.$, $\left.1.28 \times 10^{3} \mathrm{~mol}\right)$ was added. The reaction was carried out for $24 \mathrm{~h}$ at room temperature. After this, the insoluble solid of triethylammonium chloride was removed by filtration on a glass frit. The organic phase was evaporated to obtain the crude product, which was precipitated in cold $\mathrm{MeOH}$. After methanol removal, the solid was dried in vacuo. (see Supplementary Material for FT-IR analysis).

\section{An example for synthesis of $\mathrm{Ph}_{7} \mathrm{POSS}-\mathrm{OMe}_{2} \mathrm{SiVi}$ (3-Ph-OSi- Vi)}

$\mathrm{Ph}_{7}\left(\mathrm{Si}_{8} \mathrm{O}_{12}\right)(\mathrm{OH})(\mathbf{1}-\mathbf{P h}-\mathbf{O H})\left(0.50 \mathrm{~g}, 0.51 \times 10^{3} \mathrm{~mol}\right)$, anhydrous THF $\left(10 \mathrm{~cm}^{-3}\right)$ and $\mathrm{Et}_{3} \mathrm{~N} \quad\left(0.22 \mathrm{~cm}^{-3}\right.$, $\left.1.54 \times 10^{3} \mathrm{~mol}\right)$ were added to a two-neck round-bottom flask equipped with a magnetic stirrer. The reaction mixture was placed in an ice-water bath and purged with highly pure argon. Chloro(dimethyl)vinylsilane $\left(0.10 \mathrm{~cm}^{-3}\right.$, $0.77 \times 10^{3} \mathrm{~mol}$ ) was then added. The reaction was carried out for $24 \mathrm{~h}$ at room temperature. Next, the insoluble solid of triethylammonium chloride was removed by filtration on a glass frit. The organic phase was evaporated to obtain the crude product that was precipitated in cold $\mathrm{MeOH}$. After methanol removal, the solid was dried in vacuo.

Detailed experimental procedures for obtaining of hex5-enyl- and dec-9-enylchlorosilane derivatives (2m and $\mathbf{2 m - O S i )}$ as well as analytical data of all isolated compounds ( 3 and 3-OSi) and TG and DTG analysis are presented in Supplementary Material.
A general suggestion for the synthesis of both $\mathbf{3}$ and 3OSi is that after solvent evaporation there are two possible ways to purify the remaining crude products. For inert groups $\mathrm{R}=\mathrm{iBu}, \mathrm{Cy}, \mathrm{Ph}$ it is best to purify them by a precipitation from DCM in cold methanol $(\mathrm{R}=\mathrm{iBu}, \mathrm{Cy}$, $\mathrm{Ph})$ or $n$-hexane $(\mathrm{R}=\mathrm{Ph})$. Because of better solubility of products with inert groups $\mathrm{R}=\mathrm{Et}$, iOc in the abovementioned solvents, it is better to purify them by column chromatography followed by washing with cold methanol. It should be noted that presence of $-\mathrm{OSiMe}_{2}-$ spacer in 3OSi affects the inconsiderably higher solubility of these compounds in comparison with 3. Additionally, the presence of longer alkenyl chain also improves their solubility. Further spectroscopic characterization of the pure products confirmed their identity and purity.

\section{Results and discussion}

Our main interest was to synthesize a series of monoalkenylsubstituted POSS compounds varying not only in the aspect of the length of the alkenyl group at the silsesquioxane core but also the type of inert groups at seven $\mathrm{Si}$ vertexes. These two modifications not only affect differences in the synthetic procedure of certain silsesquioxanes, but also their physical properties, i.e., solubility, the state of matter, and their thermal behavior. As far as we are concerned, there is no existing scientific research of this kind. Thus, collecting the abovementioned aspects of monoalkenylPOSS compounds could be very useful for material scientists, e.g., use as comonomers for diverse applications.

We divided our experimental studies into two parallel paths which concern the synthesis of monoalkenylPOSS compounds without a $-\mathrm{OSiMe}_{2}-$ spacer (3) (Scheme 1) between alkenyl moiety and the POSS core with this spacer (3-OSi) (Scheme 2). It involved using either incompletely condensed silsesquioxane trisilanols $\mathrm{R}_{7} \mathrm{Si}_{7} \mathrm{O}_{9}(\mathrm{OH})_{3}$ (13OH) or its cubic monosilanol form $\mathrm{R}_{7} \mathrm{Si}_{8} \mathrm{O}_{12}(\mathrm{OH})$ (1$\mathbf{O H})$. Naturally, the first step was to perform the synthesis of title compounds via the hydrolytic condensation of trisilanol (1-3OH) or monosilanol (1-OH) with respective alkenyltrichlorosilane or alkenylchlorodimethylsilane (2). The general synthetic concept for condensation reaction of silanols with chlorosilanes is documented, but we performed additional studies on the impact of type of solvent, base and the stoichiometry of the reagents, etc., on the overall efficiency of the process [31-33].

\section{Synthesis of product type 3}

The "corner capping" process was optimized to enable total conversion of trisilanol 1-3OH $(\mathrm{R}=\mathrm{Et}, \mathrm{iBu}, \mathrm{iOc}, \mathrm{Cy}, \mathrm{Ph})$. 
Scheme 1 The synthetic route for obtaining monoalkenylPOSS (3) compounds
Scheme 2 The synthetic route for obtaining monoalkenylsiloxyPOSS (3OSi) compounds
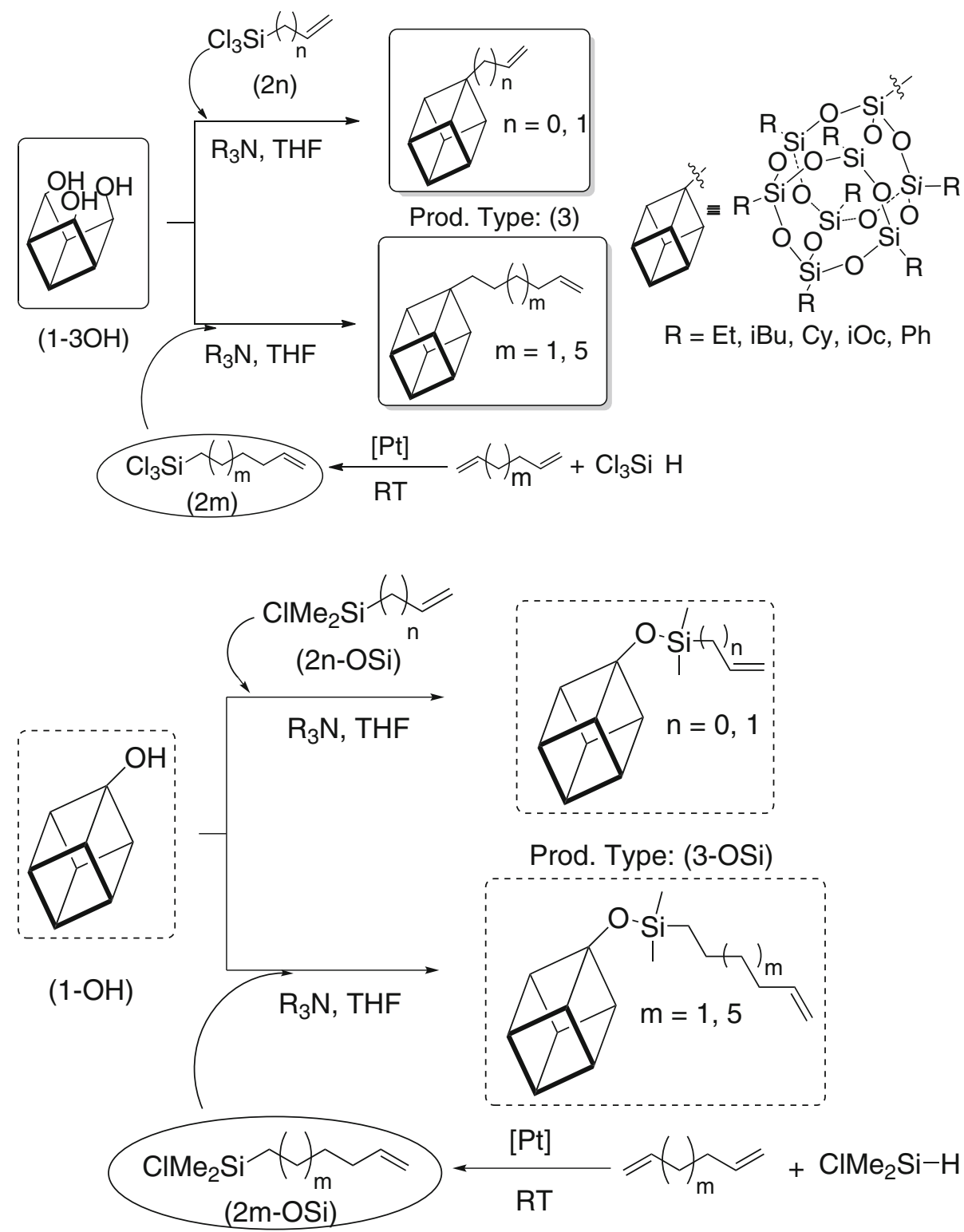

The general reaction path for synthesis of $\mathbf{3}$ is presented on Scheme 1.

The optimized condensation reaction conditions involved a series of parameters.

- Type of solvent and its amount THF, pentane or hexane were selected for tests and all of them gave similar, positive results. For 1-Ph-3OH, only THF could be used because of the solubility of the trisilanol (for 1-Ph3OH THF was used for all further studies). The reaction time was dependent on the silanol concentration in a solvent (best results for $0.03-0.05 \mathrm{M}$ ) and was monitored by FT-IR, allowing observation of disappearance of the bands assigned to $\mathrm{Si}-\mathrm{OH}$ moieties, usually $24 \mathrm{~h}$ (see Supplementary Material. Par. 1.2).
- Silane reagent For vinyl- and allylPOSS compounds the silane was commercially available, but for hex-5-enyland dec-9-enylPOSS we used pre-functionalized trichlorosilanes $(\mathbf{2 m})$ obtained via $\left[\mathrm{Pt}_{2}(\mathrm{dvs})_{3}\right]$ catalyzed hydrosilylation of 1,5-hexadiene and 1,9-decadiene, respectively, by $\mathrm{Cl}_{3} \mathrm{SiH}$ [34]. The reactions were performed at room temperature and in the excess of the corresponding diene that played the role of the reagent and reaction medium (due to ease of isolation procedure). The reaction conditions and catalyst enable high selectivity and yields of the desired $\beta$-hydrosilylation product (see Supplementary Material. Paragraph 1.1, Scheme S1). For the effective product formation, the respective trichlorosilane ( $2 \mathbf{n}$ or $\mathbf{2 m}$ ) was used in 1.01 equiv. per $1 \mathrm{~mol}$ of $\mathbf{1 - 3 0 H}$. 
- Type of $\mathrm{HCl}$ scavenger A number of compounds were tested, e.g., $\mathrm{K}_{2} \mathrm{CO}_{3}, \mathrm{~N}(i \mathrm{Pr})_{2} \mathrm{Et}$ (DIPEA) and $\mathrm{NEt}_{3}$ (TEA) and the latter was found to be the most efficient. The amount of amine was calculated for 1.5 equiv. per one $\mathrm{Si}-\mathrm{OH}$ moiety. However, in the case of phenyl trisilanol (1-Ph-3OH) the TEA was not sufficient to form the desired 3-Ph. It is probable that the basicity of amine is important and affects its possibility to react with the evolving $\mathrm{HCl}$. The more basic amines, i.e., $\mathrm{N}$ $(i \text { Pr })_{2}$ Et $\left(\right.$ DIPEA $\mathrm{pK}_{\mathrm{a}}=11.4$, TEA $\left._{\mathrm{pK}}=\mathrm{S} 10.7\right)$ enable acquisition of high yields of final products (3).

As a result, we developed a procedure for effective corner capping of 1-Ph-3OH with respective silane, which was performed in THF and with the use of DIPEA. The inert groups did not affect changes in the synthetic methodology.

In the above-optimized conditions, we investigated the scope of this reaction, using various trisilanols $(\mathbf{1 - 3 0 H}$, $\mathrm{R}=\mathrm{Et}, \mathrm{iBu}, \mathrm{iOc}, \mathrm{Cy}, \mathrm{Ph}$ ) and trichlorosilanes (2n and $\mathbf{2 m}$ ), respectively. These results are summarized in Table 1.

\section{Synthesis of product type 3-OSi}

The condensation reaction for obtaining monoalkenylsiloxyPOSS compounds (3-OSi), i.e., with the $-\mathrm{OSiMe}_{2}-$ was verified and optimized analogously to the procedure described above for 3 . The general reaction path for the synthesis of 3-OSi is presented in Scheme 2.

The presence of the siloxy linkage required using respective monosilanol $\mathbf{1 - O H}$ with five different inert substituents ( $\mathrm{R}=\mathrm{Et}, \mathrm{iBu}, \mathrm{iOc}, \mathrm{Cy}, \mathrm{Ph})$. Again, the vinyland allylsiloxyPOSS compounds were synthesized using commercially available chlorodimethylsilanes (2n-OSi), but for hex-5-enyl- and dec-9-enylPOSS we used prefunctionalized chlorodimethylsilanes (2m-OSi) obtained via $\left[\mathrm{Pt}_{2}(\mathrm{dvs})_{3}\right]$ catalyzed hydrosilylation of 1,5 -hexadiene and 1,9-decadiene by $\mathrm{ClMe}_{2} \mathrm{SiH}$, respectively (see Supplementary Material. Par. 1.1). The first step was a synthesis of respective monosilanol (1-OH), which precedes via standard condensation reaction conditions with $\mathrm{SiCl}_{4}$, followed by hydrolysis of the $\mathrm{Si}-\mathrm{Cl}$ bond, described earlier [31-33]. However, during synthesis of 1-Ph-OH, we encountered some difficulties employing known hydrolysis conditions [35] (the acidic conditions seemed to favor

Table 1 Results of condensation reaction ${ }^{\mathrm{a}}$ to obtain monoalkenylPOSS (3) compounds and their thermal properties (measured in $\mathrm{N}_{2}$ )

\begin{tabular}{|c|c|c|c|c|c|}
\hline \multirow[t]{2}{*}{ Prod. No } & \multirow[t]{2}{*}{ Isolated yield $/ \%$} & \multicolumn{2}{|c|}{ Mass loss temperature $/{ }^{\circ} \mathrm{C}$} & \multirow[t]{2}{*}{ Decomposition temperature $T_{\text {onset }} /{ }^{\circ} \mathrm{C}$} & \multirow[t]{2}{*}{ Residue at $1000^{\circ} \mathrm{C} / \%$} \\
\hline & & $T_{\mathrm{d}}^{5 \%}$ & $T_{\mathrm{d}}^{10 \%}$ & & \\
\hline 3-Et-Vi & 90 & 205 & 219 & 223 & 13 \\
\hline 3-iBu-Vi & 98 & 239 & 272 & 217 & 42 \\
\hline 3-iOc-Vi & 97 & 343 & 366 & 368 & 24 \\
\hline 3-Cy-Vi & 97 & 350 & 382 & 326 & 49 \\
\hline 3-Ph-Vi & $81^{\mathrm{b}}$ & 437 & 462 & 421 & 55 \\
\hline 3-Et-All & 96 & 203 & 214 & 207 & 16 \\
\hline 3-iBu-All & 96 & 242 & 256 & 254 & 16 \\
\hline 3-iOc-All & 96 & 319 & 351 & 359 & 15 \\
\hline 3-Cy-All & 98 & 359 & 409 & 332 & 65 \\
\hline 3-Ph-All & $81^{\mathrm{b}}$ & 439 & 460 & 441 & 63 \\
\hline 3-Et-Hex & 89 & 207 & 224 & 226 & 20 \\
\hline 3-iBu-Hex & 96 & 247 & 265 & 259 & 19 \\
\hline 3-iOc-Hex & 91 & 294 & 331 & 328 & 26 \\
\hline 3-Сy-Hex & 93 & 432 & 463 & 451 & 40 \\
\hline 3-Ph-Hex & $76^{\mathrm{b}}$ & 453 & 476 & 440 & 54 \\
\hline 3-Et-Dec & 94 & 219 & 237 & 237 & 12 \\
\hline 3-iBu-Dec & 96 & 271 & 292 & 285 & 17 \\
\hline 3-iOc-Dec & 92 & 319 & 349 & 335 & 20 \\
\hline 3-Cy-Dec & 96 & 353 & 414 & 297 & 42 \\
\hline 3-Ph-Dec & $75^{\mathrm{b}}$ & 391 & 441 & 370 & 52 \\
\hline
\end{tabular}

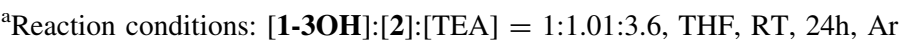

${ }^{\mathrm{b}}[\mathbf{1 - 3 O H}]:[\mathbf{2}]:[\mathrm{DIPEA}]=1: 1.01: 5$ 
intercondensation of two 1-Ph-OH molecules, which was verified by ${ }^{29} \mathrm{Si}$ NMR: - $\left.68 \mathrm{ppm}\right)$. Instead, we applied modified condensation procedure using DIPEA instead of TEA and, interestingly here, the condensation of 1-Ph3OH with $\mathrm{SiCl}_{4}$ was followed by subsequent hydrolysis of the resulting $\mathrm{Si}-\mathrm{Cl}$ bond only during extraction, in a onepot procedure. In the next step, the condensation of 1-OH with the respective chlorosilane proceeded smoothly, applying TEA as amine, without any differences with regard to the inert group type present $(\mathrm{R}=\mathrm{Et}, \mathrm{iBu}, \mathrm{iOc}$, $\mathrm{Cy}, \mathrm{Ph}$ ). It should be noted that synthesis of hex-5-enyland dec-9-enylPOSS derivatives (both types: 3 and 3-OSi) could be also performed in another way. The silsesquioxanes with $\mathrm{Si}-\mathrm{H}$ reactive bond in the POSS core or are attached via $-\mathrm{OSiMe}_{2}-$ moiety could be a hydrosilylating agent for 1,5-hexadiene and 1,9-decadiene. We have tested this option and when the $\mathrm{Si}-\mathrm{H}$ is embedded in the core, the steric hindrance of the reagent unfavors the hydrosilylation and the final products yields are low (the reaction mixture contains unreacted POSS substrate). Additionally, to avoid diene hydrosilylation from both sides, a substantial excess of diene should be applied and this solves problems concerning product isolation. As a result, the condensation procedure is more efficient for obtaining the desired products.

In the above-optimized conditions, we investigated the scope of this reaction, using various monosilanols (1-OH, $\mathrm{R}=\mathrm{Et}$, iBu, iOc, $\mathrm{Cy}, \mathrm{Ph}$ ) with monochlorosilanes (2n-OSi and $\mathbf{2 m - O S i}$ ), respectively. These results are summarized in Table 2.

All obtained products are air-stable solids (only for the iOc inert group they are viscous liquids) and can be synthesized on a multigram scale. For alkyl inert groups, they are soluble in organic solvents like $\mathrm{DCM}, \mathrm{CHCl}_{3}, \mathrm{THF}$, hexane, and toluene, but insoluble in, e.g., methanol, $\mathrm{MeCN}$. The phenyl inert groups affect the solubility; not only the silanols (1-Ph-3OH and 1-Ph-3OH), but also resulting products (3-Ph and 3-OSi-Ph) and they are insoluble in methanol, $\mathrm{MeCN}$, and hexane.

\section{Thermal analysis of monoalkenyl-(3) and monoalkenylsiloxyPOSS (3-OSi) compounds}

Subsequently, all prepared samples of $\mathbf{3}$ and 3-OSi were analyzed in terms of the influence of their structure (the type of inert functional groups as well as the chain length of the alkenyl group and the presence of $-\mathrm{OSi}\left(\mathrm{Me}_{2}\right)-$ spacer) on their thermal stability. The influence of the abovementioned structural parameters on thermal properties of measured samples were evaluated on the basis of the $5 \%\left(T_{\mathrm{d}}^{5 \%}\right)$ and $10 \%\left(T_{\mathrm{d}}^{10 \%}\right)$ mass loss temperatures, the amount of residue at $995{ }^{\circ} \mathrm{C}$ and the temperature of the main decomposition step ( $\left.T_{\text {onset }}\right)$. The details of the thermogravimetric analysis evaluated on the basis of TG curves are summarized in Tables 1 and 2. All collected thermograms are available in the Supplementary Material.

Despite the alkenyl group chain length and the presence or absence of $-\mathrm{OSi}\left(\mathrm{Me}_{2}\right)$ - moiety in the structure, the most significant influence on the thermal stability of the measured samples had the type of seven inert groups present in the silsesquioxane cage corners. It was observed that the thermal stability of the compounds increased in a row: $\mathrm{Et}<\mathrm{iBu}<\mathrm{iOc}<\mathrm{Cy}<\mathrm{Ph}$. It was abbreviated by the increase of the 5 and $10 \%$ mass loss temperatures as well as the temperatures of start and maximum degradation rate. Furthermore, the observed amount of residue at $995{ }^{\circ} \mathrm{C}$ increased in a similar way, with the exception of $\mathrm{iOc}$ derivatives. This dependency can be easily observed in the exemplary set of TG curves of hex-5-enyl-derived silsesquioxane series, presented in Fig. 2.

In all cases, the value of residue observed for $\mathrm{iOc}$ derivatives was smaller than those observed for corresponding $\mathrm{iBu}$ derivatives. This can be attributed to the higher molecular weight of iOc group in comparison with $\mathrm{iBu}$ moiety and their similar thermal stability arising from the similar (alkyl) structure. Obviously, the predominant influence of the type of inert functional groups on the thermal stability of considered compounds family is related to the stoichiometric ratio between inert and reactive groups of 7 to only 1 .

The observed impact of the type of alkenyl group of different $\mathrm{C}-\mathrm{C}$ chain length directly bonded to the $\mathrm{Si}-\mathrm{O}-\mathrm{Si}$ skeleton on the thermal stability of investigated derivatives was rather weak and more complex. There was no direct correlation between the $T_{\mathrm{d}}^{5 \%}, T_{\mathrm{d}}^{10 \%}$ and $T_{\text {onset }}$ temperatures and the alkenyl group length. However, it is worth noting that in all cases the residue observed at $995{ }^{\circ} \mathrm{C}$ was the highest for vinyl-derived silsesquioxanes and decreased with the increment of the number of carbon atoms in the alkenyl group. This can be clearly observed in the series of heptacyclohexyl silsesquioxane derivatives (see Fig. 3).

It was also observed that the incorporation of dimethylsiloxy moiety into silsesquioxane structure significantly affect their thermal properties. The onset temperatures of main decomposition step observed for derivatives with alkenyl group bonded to the silsesquioxane core through the $-\mathrm{SiO}\left(\mathrm{Me}_{2}\right)$ - bridge were, in general, higher than those observed for corresponding derivatives without this bridge. Moreover, for ethyl, isobutyl and isooctyl derivatives the values of onset temperature significantly increased with the growing alkenyl chain length while for more thermally stable derivatives (cyclohexyl and phenyl ones) this trend was reversed and the decrease in onset temperatures was observed along with the increment of the $\mathrm{C}-\mathrm{C}$ chain length of the reactive group. 
Table 2 Results of condensation reaction ${ }^{\text {a }}$ to obtain monoalkenylsiloxyPOSS (3-OSi) compounds and their thermal properties

\begin{tabular}{|c|c|c|c|c|c|}
\hline \multirow[t]{2}{*}{ Prod. No } & \multirow[t]{2}{*}{ Isolated yield/\% } & \multicolumn{2}{|c|}{ Mass loss temperature $/{ }^{\circ} \mathrm{C}$} & \multirow[t]{2}{*}{ Decomposition temperature $T_{\text {onset }} /{ }^{\circ} \mathrm{C}$} & \multirow[t]{2}{*}{ Residue at $1000{ }^{\circ} \mathrm{C} / \%$} \\
\hline & & $T_{\mathrm{d}}^{5 \%}$ & $T_{\mathrm{d}}^{10 \%}$ & & \\
\hline 3-Et-OSi-Vi & 94 & 194 & 213 & 205 & 42 \\
\hline 3-iBu-OSi-Vi & 93 & 246 & 267 & 225 & 48 \\
\hline 3-iOc-OSi-Vi & 91 & 300 & 331 & 307 & 30 \\
\hline 3-Cy-OSi-Vi & 95 & 394 & 440 & 442 & 53 \\
\hline 3-Ph-OSi-Vi & $94^{\mathrm{b}}$ & 414 & 513 & 506 & 60 \\
\hline 3-Et-OSi-All & 92 & 188 & 205 & 207 & 14 \\
\hline 3-iBu-OSi-All & 95 & 234 & 252 & 255 & 20 \\
\hline 3-iOc-OSi-All & 91 & 290 & 327 & 310 & 31 \\
\hline 3-Cy-OSi- All & 96 & 404 & 451 & 452 & 49 \\
\hline 3-Ph-OSi-All & $94^{\mathrm{b}}$ & 390 & 424 & 371 & 47 \\
\hline 3-Et-OSi-Hex & 89 & 246 & 264 & 243 & 32 \\
\hline 3-iBu-OSi-Hex & 95 & 226 & 260 & 259 & 24 \\
\hline 3-iOc-OSi-Hex & 94 & 306 & 354 & 380 & 16 \\
\hline 3-Cy-OSi-Hex & 95 & 314 & 367 & 441 & 40 \\
\hline 3-Ph-OSi-Hex & $96^{\mathrm{b}}$ & 271 & 395 & 410 & 44 \\
\hline 3-Et-OSi-Dec & 89 & 225 & 261 & 255 & 22 \\
\hline 3-iBu-OSi-Dec & 95 & 275 & 297 & 293 & 18 \\
\hline 3-iOc-OSi-Dec & 93 & 285 & 356 & 397 & 16 \\
\hline 3-Cy-OSi-Dec & 94 & 311 & 399 & 448 & 35 \\
\hline 3-Ph-OSi-Dec & $95^{\mathrm{b}}$ & 213 & 335 & 409 & 28 \\
\hline
\end{tabular}

${ }^{a}$ Reaction conditions: $[\mathbf{1 - O H}]:[\mathbf{2}]:[\mathrm{TEA}]=1: 1.01: 1.5$, THF, RT, 24h, $\mathrm{Ar}$

${ }^{\mathrm{b}}[\mathbf{1 - O H}]:[\mathbf{2}]:[\mathrm{TEA}]=1: 1.01: 3$

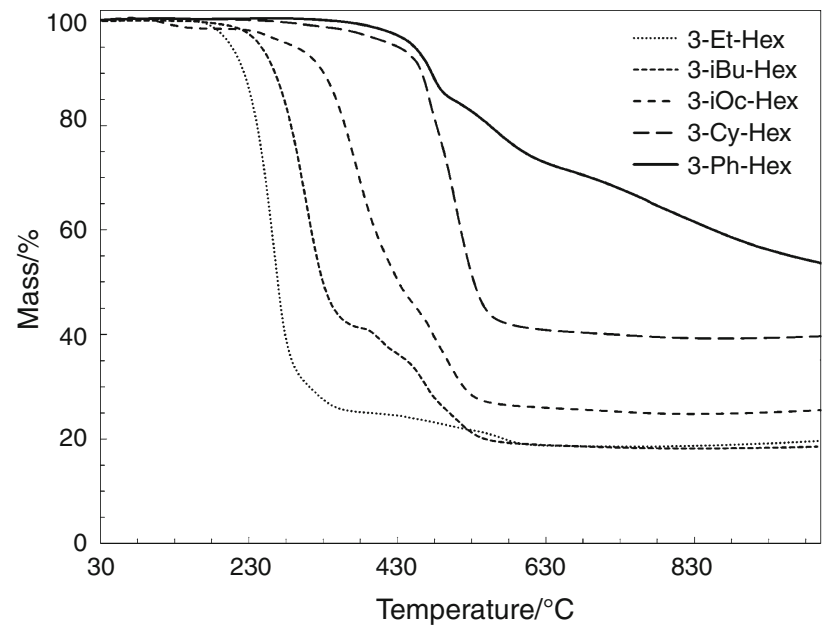

Fig. 2 TG curves of hex-5-enyl-derived silsesquioxanes bearing different inert organic groups

\section{DSC analysis of monoalkenyl-(3) and monoalkenylsiloxyPOSS (3-OSi) compounds}

The thermogravimetric studies of a described group of compounds were followed by the DSC experiments for the

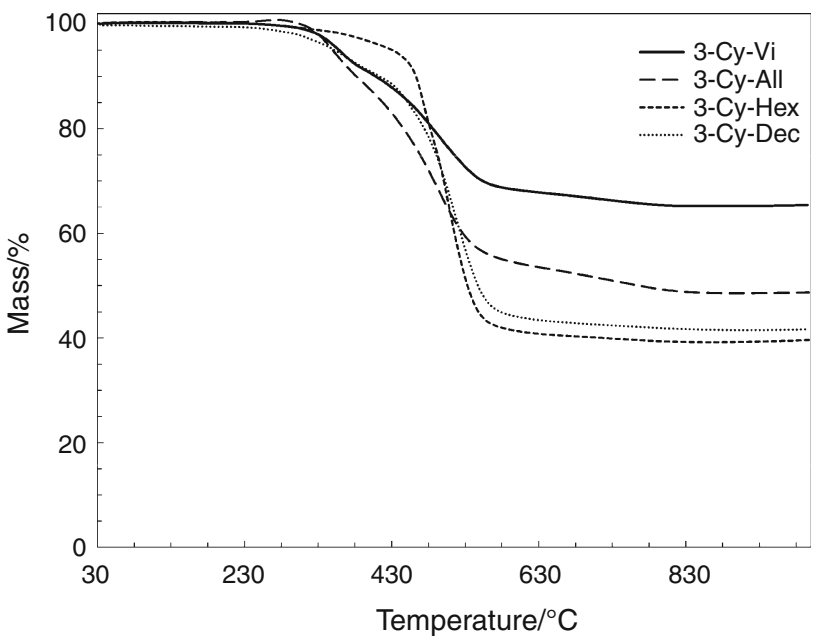

Fig. 3 TG curves of heptacyclohexyl silsesquioxanes bearing different alkenyl functional groups

determination of their melting and crystallization temperatures. The measurements were taken in a temperature range from $-80^{\circ} \mathrm{C}$ to the temperature of the beginning of sample decomposition established on the basis of TG analysis. Unfortunately, due to the technical limitations and 
samples nature, it was not possible to measure mentioned parameters for all the samples. In many cases, either the melting temperatures $\left(T_{\mathrm{m}}\right)$ of the measured samples were above their decomposition temperatures, or they melted with decomposition (e.g., phenyl derivatives), which made it impossible to get insight into any correlation between their structure and thermal properties. Nevertheless, on the basis of the partial results presented in Table 3, it can be observed that the number of carbon atoms present in the alkenyl group chain influences the melting and crystallization temperatures $\left(T_{\mathrm{c}}\right)$ of monoalkenyl-derived silsesquioxanes despite the type of inert organic groups present in their structure and the presence or absence of $\mathrm{SiO}\left(\mathrm{Me}_{2}\right)$ - bridge. The obtained results suggest that melting and crystallization temperatures decrease with the increment of alkenyl chain length. Unfortunately, the obtained data were not sufficient to determine the impact of the- $\mathrm{SiO}\left(\mathrm{Me}_{2}\right)$ - linkage on the thermal behavior of measured sampled.

Although we were not able to establish the melting and crystallization temperatures for isooctyl silsesquioxane derivatives, the performed experiment enabled us to determine the glass transition temperatures for this group of compounds. As shown in Table $4, T_{\mathrm{g}}$ temperatures decrease with an increasing amount of carbon atoms in the alkenyl group from -32 to almost $-45^{\circ} \mathrm{C}$ for vinyl and decenyl derivative, respectively. In this case, it was also possible to observe the influence of the presence of $-\mathrm{SiO}$ $\left(\mathrm{Me}_{2}\right)$ - bridge in the heptaisooctylsilsesquioxane series structure on their thermal behavior. In comparison with the "regular" silsesquioxanes, those bearing alkenyl group bonded to the $\mathrm{Si}-\mathrm{O}-\mathrm{Si}$ core through the $-\mathrm{SiO}\left(\mathrm{Me}_{2}\right)_{-}$ moiety were characterized with lower glass transition temperatures of more than $3{ }^{\circ} \mathrm{C}$.

Table 3 Melting and crystallization temperatures of selected monoalkenyl-derived silsesquioxanes

\begin{tabular}{lrr}
\hline Prod. No & $T_{\mathrm{m}} /{ }^{\circ} \mathrm{C}$ & $T_{\mathrm{c}} /{ }^{\circ} \mathrm{C}$ \\
\hline 3-Et-Hex & 110.87 & 86.40 \\
3-Et-Dec & 104.26 & 74.60 \\
3-Et-OSi-All & 88.99 & 53.73 \\
3-iBu-Hex & 180.51 & 117.67 \\
3-iBu-Dec & 113.82 & 76.49 \\
3-iBu-OSi-All & 191.98 & 186.67 \\
3-iBu-OSi-Hex & 117.29 & 98.71 \\
3-iBu-OSi-Dec & 83.56 & 51.26 \\
3-Cy-Dec & 259.19 & 244.80 \\
3-Cy-OSi-Hex & 290.87 & 281.74 \\
3-Cy-OSi-Dec & 271.12 & 228.13 \\
\hline
\end{tabular}

Table 4 Glass transition temperatures of heptaisooctylalkenyl silsesquioxane derivatives

\begin{tabular}{ll}
\hline Prod. No & $T_{\mathrm{g}} /{ }^{\circ} \mathrm{C}$ \\
\hline 3-iOct-Vi & -31.88 \\
3-iOct-All & -34.07 \\
3-iOct-Hex & -43.01 \\
3-iOct-Dec & -46.70 \\
3-iOct-OSi-Vi & -37.57 \\
3-iOct-OSi-All & -41.24 \\
3-iOct-OSi-Hex & -44.05 \\
3-iOct-OSi-Dec & -51.64 \\
\hline
\end{tabular}

Solubility tests of monoalkenyl-(3) and monoalkenylsiloxyPOSS (3-OSi) compounds with $\mathrm{iBu}$ and $\mathrm{Ph}$ inert groups

Finally, the THF, DCM and toluene solubility of selected derivatives with $\mathrm{iBu}$ and $\mathrm{Ph}$ inert groups was investigated. The resulted data are presented in Table 5.

Besides the fundamental difference in the solubility of isobutyl and phenyl silsesquioxane derivatives, a correlation between the alkenyl group chain length, the presence of the $-\mathrm{SiO}\left(\mathrm{Me}_{2}\right)-$, and the compound's solubility were all also observed. The solubility of silsesquioxanes increases despite of solvent type with a growing number of carbon atoms in the alkenyl chain. Also, the presence of the $-\mathrm{SiO}$ $\left(\mathrm{Me}_{2}\right)$ - bridge increases the solubility of $\mathrm{iBu}$ and $\mathrm{Ph}$

Table 5 Solubility of 3-iBu- and 3-Ph- compounds with different alkenyl functional groups

\begin{tabular}{llll}
\hline Prod. No & \multicolumn{3}{l}{ Solubility/g cm } \\
\cline { 2 - 4 } & DCM & THF & Toluene \\
\hline 3-iBu-Vi & 0.50 & 0.63 & 0.42 \\
3-iBu-All & 0.80 & 0.87 & 0.90 \\
3-iBu-Hex & 1.47 & 1.15 & 0.95 \\
3-iBu-Dec & 2.22 & 1.61 & 1.35 \\
3-iBu-OSi-Vi & 0.61 & 0.73 & 0.49 \\
3-iBu-OSi-All & 1.22 & 1.00 & 1.00 \\
3-iBu-OSi-Hex & 2.00 & 1.33 & 1.05 \\
3-iBu-OSi-Dec & 1.82 & 2.00 & 1.67 \\
3-Ph-Vi & 0.13 & 0.11 & $0.02^{\mathrm{a}}$ \\
3-Ph-All & 0.36 & 0.31 & $0.04^{\mathrm{a}}$ \\
3-Ph-Hex & 0.35 & 0.31 & 0.05 \\
3-Ph-Dec & 0.43 & 0.41 & 0.10 \\
3-Ph-OSi-Vi & 0.16 & 0.13 & $0.03^{\mathrm{a}}$ \\
3-Ph-OSi-All & 0.42 & 0.37 & $0.05^{\mathrm{a}}$ \\
3-Ph-OSi-Hex & 0.40 & 0.33 & 0.06 \\
3-Ph-OSi-Dec & 0.50 & 0.59 & 0.15 \\
\hline at 50 & & &
\end{tabular}


derived silsesquioxanes by approximately 20 and $10 \%$, respectively.

\section{Conclusions}

Herein, we have reported the design and synthesis of a library of monoalkenylsubstituted silsesquioxanes characterized by a rigid $\mathrm{Si}-\mathrm{O}-\mathrm{Si}$ structure and the presence of one alkenyl functional moiety with a different chain length, from vinyl to dec-9-enyl-, and five inert groups, i.e., Et, $\mathrm{iBu}, \mathrm{iOc}, \mathrm{Cy}, \mathrm{Ph}$. Efficient procedures for their synthesis were described, which are based on a condensation, "corner capping" type reaction, as well as a hydrosilylation process using respective chlorosilanes which are not all commercially available. As a result of our studies, we obtained 40 compounds, more than a half of which have not previously been described. They were thoroughly characterized by spectroscopic methods. The results of thermal analysis exhibited a strong structure-thermal properties relation. It was shown that even a subtle difference in the structure of only one of eight functional groups present in the POSS molecule can strongly affect its thermal stability, melting and crystallization behavior, and change the glass transition point. The same structural parameters impact the solubility of a described group of compounds. All of these physicochemical parameters, together with the modulated reactivity of functional groups and their accessibility (caused by different steric hindrance), can influence the properties of the final materials prepared with their use, as well as the conditions for their synthesis.

Acknowledgements Financial support from the National Science Centre (Poland) Project OPUS No. DEC-2012/07/B/ST5/03042 and National Centre for Research and Development in Poland PBS3/A1/ $16 / 2015$ is acknowledged.

Open Access This article is distributed under the terms of the Creative Commons Attribution 4.0 International License (http://crea tivecommons.org/licenses/by/4.0/), which permits unrestricted use, distribution, and reproduction in any medium, provided you give appropriate credit to the original author(s) and the source, provide a link to the Creative Commons license, and indicate if changes were made.

\section{References}

1. Cordes DB, Lickiss PD, Rataboul F. Recent developments in the chemistry of cubic polyhedral oligosilsesquioxanes. Chem Rev. 2010;110:2081-173.

2. Hartmann-Thompson C. Applications of polyhedral oligomeric silsesquioxanes. New York: Springer; 2011.

3. Zhou H, Ye Q, Xu J. Polyhedral oligomeric silsesquioxane-based hybrid materials and their applications. Mater Chem Front. 2017;1:212-30.
4. Laine RM. Nanobuilding blocks based on the $[\mathrm{OSiO} 1.5]_{x}(x=6$, 8, 10) octasilsesquioxanes. J Mater Chem. 2005;15:3725-44.

5. Kaźmierczak J, Kuciński K, Hreczycho G. Highly efficient catalytic route for the synthesis of functionalized silsesquioxanes. Inorg Chem. 2017;56:9337-42.

6. Ayandele E, Sarkar B, Alexandridis P. Polyhedral oligomeric silsesquioxane (POSS)-containing polymer nanocomposites. Nanomaterials. 2012;2:445-75.

7. Ye Q, Zhou H, Xu J. Cubic polyhedral oligomeric silsesquioxane based functional materials: synthesis, assembly, and applications. Chem Asian J. 2016;11:1322-37.

8. Lichtenhan JD, Otonari YA, Cam MJ, Carr MJ. Linear hybrid polymer building blocks: methacrylate-functionalized polyhedral oligomeric silsesquioxane monomers and polymers. Macromolecules. 1995;28:8435-7.

9. Franczyk A, He H, Burdyn J. Synthesis of high molecular weight polymethacrylates with polyhedral oligomeric silsesquioxane moieties by atom transfer radical polymerization. ACS Macro Lett. 2014;3:799-802.

10. Alexandris S, Franczyk A, Papamokos G, Marciniec B, Matyjaszewski K, Koynov K, et al. Polymethacrylates with polyhedral oligomeric silsesquioxane (POSS) moieties: influence of spacer length on packing, thermodynamics, and dynamics. Macromol Am Chem Soc. 2015;48:3376-85.

11. Shockey EG, Bolf AG, Jones PF, Schwab JJ, Chaffee KP, Haddad TS, et al. Functionalized polyhedral oligosilsesquioxane (POSS) macromers: new graftable POSS hydride, POSS $\alpha$-olefin, POSS epoxy, and POSS chlorosilane macromers and POSSsiloxane triblocks. Appl Organomet Chem. 1999;13:311-27.

12. Zhang W, Camino G, Yang R. Polymer/polyhedral oligomeric silsesquioxane (POSS) nanocomposites: an overview of fire retardance. Prog Polym Sci. 2017;67:77-125.

13. Fu BX, Lee A, Haddad TS. Styrene-butadiene-styrene triblock copolymers modified with polyhedral oligomeric silsesquioxanes. Macromolecules. 2004;37:5211-8.

14. Ryu H-S, Kim D-G, Lee J-C. Polysiloxanes containing polyhedral oligomeric silsesquioxane groups in the side chains; synthesis and properties. Polymer. 2010;51:2296-304.

15. Zhang W, Müller A. Architecture, self-assembly and properties of well-defined hybrid polymers based on polyhedral oligomeric silsequioxane (POSS). Prog Polym Sci. 2013;38:1121-62.

16. Wojtala A. Methods of grafting of polyethylene with polar compounds. Polymer. 2002;47:694-9.

17. Li L, Liu H. Facile construction of hybrid polystyrene with a string of lantern shape from monovinyl-substituted POSS and commercial polystyrene via Friedel-Crafts reaction and its properties. RSC Adv. 2014;4:46710-7.

18. Phillips SH, Haddad TS, Tomczak SJ. Developments in nanoscience: polyhedral oligomeric silsesquioxane (POSS)polymers. Curr Opin Solid State Mater Sci. 2004;8:21-9.

19. Wu J, Haddad TS, Mather PT. Vertex group effects in entangled polystyrene-polyhedral vertex group effects in entangled polystyrene-polyhedral oligosilsesquioxane (POSS) copolymers. Macromolecules. 2009;42:1142-52.

20. Haddad TS, Lichtenhan JD. Hybrid organic-inorganic thermoplastics: styryl-based polyhedral oligomeric silsesquioxane polymers. Macromolecules. 1996;29:7302-4.

21. Li W, Chen T, Guan C, Gong D, Mu J, Chen ZR, et al. Influence of polyhedral oligomeric silsesquioxane structure on the disentangled state of ultrahigh molecular weight polyethylene nanocomposites during ethylene in situ polymerization. Ind Eng Chem Res. 2015;54:1478-86.

22. Zheng L, Kasi RM, Farris RJ, Coughlin EB. Synthesis and thermal properties of hybrid copolymers of syndiotactic polystyrene and polyhedral oligomeric silsesquioxane. J Polym Sci Part A Polym Chem. 2002;40:885-91. 
23. Zheng L, Farris RJ, Coughlin EB. Novel polyolefin nanocomposites: synthesis and characterizations of metallocene-catalyzed polyolefin polyhedral oligomeric silsesquioxane copolymers. Macromolecules. 2001;34:8034-9.

24. Seurer B, Coughlin EB. Ethylene-propylene-silsesquioxane thermoplastic elastomers. Macromol Chem Phys. 2008;209:1198-209.

25. Zhang HX, Shin YJ, Yoon KB, Lee DH. Preparation and properties of propylene/POSS copolymer with rac-Et(Ind $)_{2} \mathrm{ZrCl}_{2}$ catalyst. Eur Polym J. 2009;45:40-6.

26. Yu LY, Shen HM, Xu ZL. PVDF-TiO 2 composite hollow fiber ultrafiltration membranes prepared by $\mathrm{TiO}_{2}$ sol-gel method and blending method. J Appl Phys. 2009;113:1763-72.

27. Tsuchida A, Bolln C, Sernetz FG, Frey H, Mulhaupt R. Ethene and propene copolymers containing silsesquioxane side groups. Macromolecules. 1997;30:2818-24.

28. Groch P, Dziubek K, Czaja K, Dudziec B, Marciniec B. Copolymers of ethylene with monoalkenyl- and monoalkenyl (siloxy)silsesquioxane (POSS) comonomers-synthesis and characterization. Eur Polym J. 2017;90:368-82.

29. Groch P, Dziubek K, Czaja K, Białek M, Adamczyk-Tomiak K, Rabiej S, et al. Ethylene/POSS copolymerization behavior of postmetallocene catalysts and copolymer characteristics. J Polym Sci Part A Polym Chem. 2017;55:3918-34.
30. Anderson SE, Mitchell C, Haddad TS, Vij A, Schwab JJ, Bowers MT. Structural characterization of POSS siloxane dimer and trimer. Chem Mater. 2006;18:1490-7.

31. Lickiss PD, Rataboul F. Fully condensed polyhedral oligosilsesquioxanes (POSS): from synthesis to application. Adv Organomet Chem. 2008;57:1-116.

32. Duchateau R, Abbenhuis HCL, Van Santen RA, Thiele SK, Van Tol MFH. Half-sandwich titanium complexes stabilized by a novel silsesquioxane ligand: soluble model systems for silicagrafted olefin polymerization catalysts. Organometallics. 2009;12:5222-4.

33. Żak P, Pietraszuk C, Marciniec B, Spólnik B, Danikiewicz W. Efficient functionalisation of cubic monovinylsilsesquioxanes via cross-metathesis and silylative coupling with olefins in the presence of ruthenium complexes. Adv Synth Catal. 2009;351:2675-82.

34. Marciniec B, Maciejewski H, Pietraszuk C, Pawluć P. Hydrosilylation: a comprehensive review on recent advances. New York: Springer; 2009.

35. Seurer B, Vij V, Haddad T, Mabry JM, Lee A. Thermal transitions and reaction kinetics of polyhederal silsesquioxane containing phenylethynylphthalimides. Macromolecules. 2010;43:9337-47. 